\title{
SuperNO2VA en pacientes críticos con COVID-19 Una alternativa para el Soporte Respiratorio No Invasivo.
}

Mira Betancur RA, De Pablo Pajares A.

\section{Hospital Universitario Ramón y Cajal, Madrid.}

\section{Resumen}

La enfermedad por coronavirus 2019 (COVID-19) se conoció a finales del 2019, inicialmente en la provincia China de Wuhan, pero se extendió de manera rápida a lo largo del mundo y en Febrero de 2020 ya estaba presente en varios países de todos los continentes; a mediados de Marzo la Organización Mundial de La salud (OMS) la declaró oficialmente como pandemia. El espectro clínico abarca desde cuadros asintomáticos o con sintomatología leve hasta situaciones graves potencialmente mortales debido sobre todo a insuficiencia respiratoria aguda hipoxémica, sepsis o fracaso multiorgánico. La mayoría de pacientes graves que ingresan en la Unidad de Cuidados Críticos (UCI) requiere soporte respiratorio invasivo, no invasivo o la combinación de ambos.

La gran avalancha de pacientes que ingresaron en los hospitales de Madrid en un periodo muy corto de tiempo condicionó una presión asistencial sin precedentes en el sistema sanitario y precisó de una reorganización estructural y funcional a todos los niveles; esta gran exigencia hizo que por momentos la disponibilidad de algunos recursos resultara claramente insuficiente y fue necesario improvisar mecanismos de ayuda y soporte así como diversificar estructuras y medios incluso en sitios poco habituales; la creación de equipos de trabajo y el equipamiento de camas de UCI fue una las mayores necesidades; en este escenario la labor del servicio de Anestesiología y Reanimación resultó fundamental dada su capacidad de adaptación y la destreza probada en el soporte y manejo del paciente crítico. El soporte respiratorio no invasivo (SRNI) es una estrategia clave para el manejo del paciente con insuficiencia respiratoria aguda grave, tanto dentro como fuera de la UCI; es cierto que al principio de la pandemia su uso no fue muy extendido, primero por el temor al fallo de la técnica y segundo y más importante por el temor fundado del personal sanitario al contagio por la dispersión de aerosoles; finalmente se ha reconocido su eficacia si se aplica con la indicación adecuada, en el momento correcto y en las instalaciones apropiadas. La mascarilla SuperNO2VA es un dispositivo poco conocido hasta ahora y que en situaciones como la que enfrentamos puede resultar de gran utilidad en casos concretos.

En este artículo pretendemos describir las características clave de la neumonía por COVID-19 y el papel que juegan los sistemas de SRNI en su manejo, en concreto la mascarilla de PAP SuperNO2VA, describimos algunos casos de su uso en diversos escenarios.

\section{Introducción}

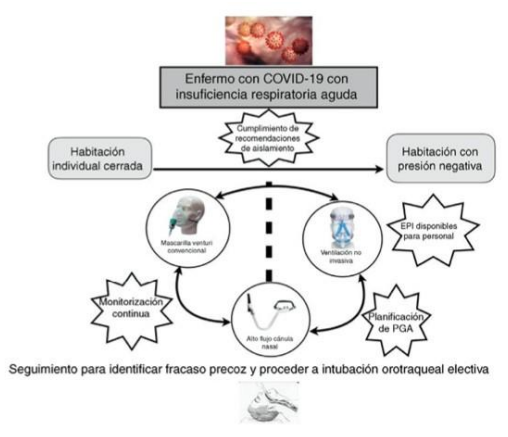

La enfermedad por coronavirus 2019 (COVID-19) se conoció a finales del 2019, inicialmente en la provincia China de Wuhan, pero se extendió de manera rápida a lo largo del mundo y en Febrero de 2020 ya estaba presente en varios países de todos los continentes; a mediados de Marzo la Organización Mundial de La salud (OMS) la declaró oficialmente como pandemia. El espectro clínico abarca desde cuadros asintomáticos o con sintomatología leve hasta situaciones 
graves potencialmente mortales debido sobre todo a insuficiencia respiratoria aguda hipoxémica, sepsis o fracaso multiorgánico. La mayoría de pacientes graves que ingresan en la Unidad de Cuidados Críticos (UCI) requiere soporte respiratorio invasivo, no invasivo o la combinación de ambos.

La gran avalancha de pacientes que ingresaron en los hospitales de Madrid en un periodo muy corto de tiempo condicionó una presión asistencial sin precedentes en el sistema sanitario y precisó de una reorganización estructural $\mathrm{y}$ funcional a todos los niveles; esta gran exigencia hizo que por momentos la disponibilidad de algunos recursos resultara claramente insuficiente y fue necesario improvisar mecanismos de ayuda y soporte así como diversificar estructuras y medios incluso en sitios poco habituales; la creación de equipos de trabajo $\mathrm{y}$ el equipamiento de camas de UCI fue una las mayores necesidades; en este escenario la labor del servicio de Anestesiología y Reanimación resultó fundamental dada su capacidad de adaptación y la destreza probada en el soporte y manejo del paciente crítico. El soporte respiratorio no invasivo (SRNI) es una estrategia clave para el manejo del paciente con insuficiencia respiratoria aguda grave, tanto dentro como fuera de la UCI; es cierto que al principio de la pandemia su uso no fue muy extendido, primero por el temor al fallo de la técnica y segundo y más importante por el temor fundado del personal sanitario al contagio por la dispersión de aerosoles; finalmente se ha reconocido su eficacia si se aplica con la indicación adecuada, en el momento correcto y en las instalaciones apropiadas. La mascarilla SuperNO2VA es un dispositivo poco conocido hasta ahora y que en situaciones como la que enfrentamos puede resultar de gran utilidad en casos concretos.
En este artículo pretendemos describir las características clave de la neumonía por COVID-19 y el papel que juegan los sistemas de SRNI en su manejo, en concreto la mascarilla de PAP SuperNO2VA, describimos algunos casos de su uso en diversos escenarios.

El 16 de marzo de 2020 la organización mundial de la salud (WHO) declaró como pandemia la infección causada por el SARS-CoV-2 (Severe acute respiratory syndrome Coronavirus 2) (1).

Después de EEUU, Rusia, Brasil y Reino Unido, España ha sido el quinto país con un mayor número de casos confirmados hasta el momento; el primer caso en el país se identificó a finales de enero. En Madrid, un área de casi 7 millones de habitantes donde la epidemia ha tenido el mayor impacto se conoció el primer caso exactamente el 25 de febrero; en la actualidad el número de casos confirmados en todo el país es de casi 250.000 , se documentan más de 28.000 fallecidos y aproximadamente 150.000 recuperados de la enfermedad; al rededor del $30 \%$ de estas cifras corresponden a Madrid (2,3).

La progresión de la pandemia en la capital española es similar a la observada en las áreas más afectadas en los países occidentales, como es el caso de Lombardía en Italia, Sao Pablo en Brasil o de New York en USA. Los sistemas sanitarios de estas áreas han sufrido una presión intensa casi llegando al colapso en algún momento con unas tasas de ingresos y mortalidad altísimas (por 100 mil habitantes): 132 en Lombardía, 140 en NY y 190 en Madrid (4).

Al momento del inicio de la pandemia el curso de la enfermedad resultó desconcertante a la vez que devastador para los servicios médicos. Al tratarse 
de un curso clínico incierto y de fisiopatología no muy bien conocida, las opciones terapéuticas ofrecidas a los pacientes no presentaban una evidencia probada ni la eficacia esperada; además la disparidad regional en la disponibilidad y accesibilidad de los recursos de atención en los diferentes países afectados, y entre regiones de cada país impidió un abordaje consensuado de su manejo (5). Si bien se ha documentado que la mayoría de las personas que contraen el virus no desarrollan ninguna clínica o sólo una sintomatología leve, los resultados de los diversos análisis preliminares han mostrado que hasta un $15 \%$ de los infectados desarrollaron un tipo grave de la enfermedad con necesidad de ingreso $y$ de soporte con oxigenoterapia; a su vez entre un 3-5\% necesitan ingreso en UCI precisando de asistencia respiratoria invasiva, no invasiva o ambas (6).

Parece claro que el curso clínico y la fisiopatología del proceso infeccioso cuando es grave implica el desarrollo de un síndrome complejo de respuesta inflamatoria sistémica llevando incluso al daño de varios órganos y sistemas (fracaso multiorgánico) y complicaciones graves de tipo trombótico en todos los órganos, pero la insuficiencia Respiratoria Aguda (IRA) sobre todo de origen hipoxémico es la principal causa de muerte.

\section{Características de la Neumonía por COVID-19}

El proceso fisiopatológico de la IRA por COVID es complejo y en un principio resultó difícil de comprender. Se identifican tres fases o estadios bien diferenciados: fase I o de infección precoz, fase II o de afectación pulmonar severa $y$ fase III $o$ de respuesta inflamatoria. Tras la primoinfección (fase I) se produce una gran replicación viral y la sintomatología suele ser entre leve - moderada consistente en fiebre, tos seca, cefalea $y$ en ocasiones acompañada de síntomas gastrointestinales como dolor abdominal, vómitos y diarrea; pasada aproximadamente 1 semana el cuadro empieza a empeorar ya con disnea, taquipnea y desaturación progresivas (fase II); y por último en algunos pacientes se desarrolla la fase III $o$ inflamatoria mediada por macrófagos y citoquinas que desencadenan una inflamación sistémica y en concreto a nivel pulmonar que puede llegar a ser severa y desencadenar la muerte del paciente (7).

Se produce un distrés (SDRA) tremendamente hipoxémico e hipercápnico, debido en primer lugar a la respuesta inflamatoria secundaria a la activación de macrófagos $y$ citoquinas; esto ocasiona un proceso intersticial difuso con gran alteración de la relación ventilación-perfusión e inhibición del mecanismo compensatorio de la vasoconstricción pulmonar hipóxica ahondando más la hipoxemia; habitualmente no existen grandes áreas de atelectasias pulmonares (salvo en pacientes obesos) y al principio la complianza pulmonar suele estar conservada, con lo cual el tamaño pulmonar funcional no suele estar muy comprometido (8). $\mathrm{Si}$ el proceso continúa puede desencadenar en fibrosis pulmonar, limitando su capacidad de intercambio y llevando a la muerte del paciente.

Para el manejo y el soporte respiratorio existen una serie de mecanismos que permiten aumentar el aporte de oxígeno a los pacientes, q van desde la oxigenoterapia convencional hasta el soporte avanzado con membrana de oxigenación extracorpórea (ECMO), pasando por diversos modos intermedios de asistencia ventilatoria 
tanto invasiva (VMI) como no invasiva (VMNI) (9).

El objetivo primordial es restablecer la oxigenación, y esto lo conseguimos de dos formas: aumentando la Fracción inspirada de Oxígeno (FiO2) o aumentando la presión en la vía aérea.

\section{INDICE DE OXIGENACION= Presión media de la vía aérea $x$ FiO2 x 100 / PaO2}

De esta fórmula se deduce que una vez alcanzada la máxima $\mathrm{FiO} 2$ que podemos aportar, la única alternativa para mejorar la oxigenación es aumentar la presión media en la vía aérea, y esto lo podemos conseguir a través de la PEEP o CPAP (10).

El uso de CPAP mediante técnicas de soporte no invasivo durante la pandemia no ha recibido la atención ni la trascendencia que quizá podría haber generado sobre todo al principio básicamente por la falta de dispositivos adecuados y suficientes en un momento de gran demanda, también por la falta de tiempo necesario para dedicar a la aplicación y adecuación del dispositivo debido a la presión asistencial por la avalancha de pacientes casi incontrolable y por último, y no menos importante, por el temor fundado del personal sanitario a contagiarse por la alta probabilidad de generar aerosoles; es preciso recordar que España tiene el poco honroso récord de personal sanitario contagiado con cifras que sobrepasan los 50.000 hasta el momento.

Los criterios utilizados para iniciar el soporte respiratorio invasivo o no invasivo son bastante homogéneos según las diferentes guías:

- Criterios clínicos:

- Disnea moderada-grave con signos de trabajo respiratorio $\mathrm{y}$ uso de la musculatura accesoria

o movimiento abdominal paradójico.

- Taquipnea mayor de $30 \mathrm{rpm}$.

- Alteración del nivel de conciencia secundario al fallo respiratorio.

- Criterios gasométricos:

- $\mathrm{PaO} 2 / \mathrm{FiO} 2<200$ o la necesidad de administrar una FiO2 superior a 0,4 para conseguir una $\mathrm{SpO} 2$ de al menos 92\%.

- Fallo ventilatorio agudo $(\mathrm{pH}<7,25 \quad$ con $\quad \mathrm{PaCO} 2>45$ $\mathrm{mmHg}$ ).

En pacientes sin ninguno de estos criterios el tratamiento indicado inicialmente sería la oxigenoterapia convencional (11).

El uso de sistemas de soporte respiratorio no invasivo ha sido objeto de una amplia difusión y evolución en los últimos años como alternativa y/o complemento a la ventilación mecánica invasiva en el manejo de una amplia gama de patologías que cursa con insuficiencia respiratoria aguda $\mathrm{o}$ crónica.

En el manejo de la IRA por COVID su uso ha resultado controvertido, principalmente por que las tasas de fracaso encontradas en las diversas series publicadas hasta ahora es hasta del $30 \%$ y sobre todo por la posibilidad de generar aerosoles lo que incrementa el riesgo de transmisión e infección del personal sanitario que manipula los dispositivos y se expone a un contacto extremadamente cercano a la vía aérea del paciente (12).

De hecho en muchas publicaciones $\mathrm{y}$ conferencias de consenso iniciales no recomendaban el uso de esta clase de dispositivos $(13,14)$ salvo en casos muy seleccionados y siempre que se cumplan los siguientes criterios: 
- Pacientes sin patología previa (IRA de novo) con insuficiencia respiratoria hipoxémica y susceptibles de escalar hasta la ECMO de ser necesario. Es requisito esencial que exista un equipo experto, una adecuada monitorización y un protocolo que identifique de manera precoz el posible fallo de la técnica (Índice de ROX, índice de HACOR, etc), y no se retrase la intubación orotraqueal (IOT) cuando sea necesaria.

- Pacientes con fracaso hipoxémico grave, con orden de no IOT y techo terapéutico en la VMNI.

- Pacientes con exacerbación grave de EPOC con fallo respiratorio hipercápnico agudo o agudizado.

Siempre deben estar aseguradas las medidas de protección de todo el personal sanitario que atiende a pacientes con sospecha o confirmación de infección por COVID-19 en tratamiento con Soporte Respiratorio No Invasivo (SRNI) y deben llevar un equipo de protección individual (EPI) completo y adecuado que incluya una mascarilla de alta eficacia FFP2 o preferiblemente FFP3, gafas de protección integral, gorro, guantes, calzas y batas impermeables (15).

Sería ideal poder ubicar al paciente en habitaciones individuales con presión negativa y hacer una adecuada selección del dispositivo, su configuración, elección del tipo de interfase y la mejor optimización de los diferentes parámetros de asistencia.

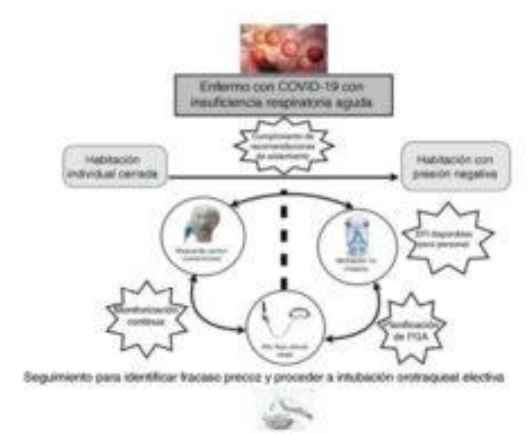

Figura 1. Recomendaciones de aislamiento y seguridad de la OMS

Los Dispositivos de SRNI utilizados en nuestro centro durante la pandemia y con potencial capacidad para generar CPAP son:

- Gafas Nasales de Alto Flujo.

- VMNI con interfase nasal, oronasal y totalface.

- Diversos sistemas de CPAP (Máscaras Decathlon, SuperNO2VA, Circuitos Mapleson tipo $\mathrm{C}$ con mascarillas de anestesia y arneses), con válvulas APL y de resistencia espiratoria gradual.

\section{SuperNO2VA (Vyaire Mettawa, IL).}

Medical,

El dispositivo nasal de ventilación a presión positiva SuperNO2VA está concebido para usarse en periodos cortos de tiempo $(<24 \mathrm{~h})$ en pacientes adultos $(>30 \mathrm{~kg})$ durante el proceso de sedaciones o anestesia (16).

Existen publicadas series de casos en procesos de sedación para realizar endoscopias, fibrobroncoscopias, ecocardiografía transesofágica y como medida de preoxigenación, preintubación o de soporte postextubación en pacientes obesos $\mathrm{o}$ con otras patologías complejas. También para el manejo de angioedema y espasmo glótico $(17,18$,). 


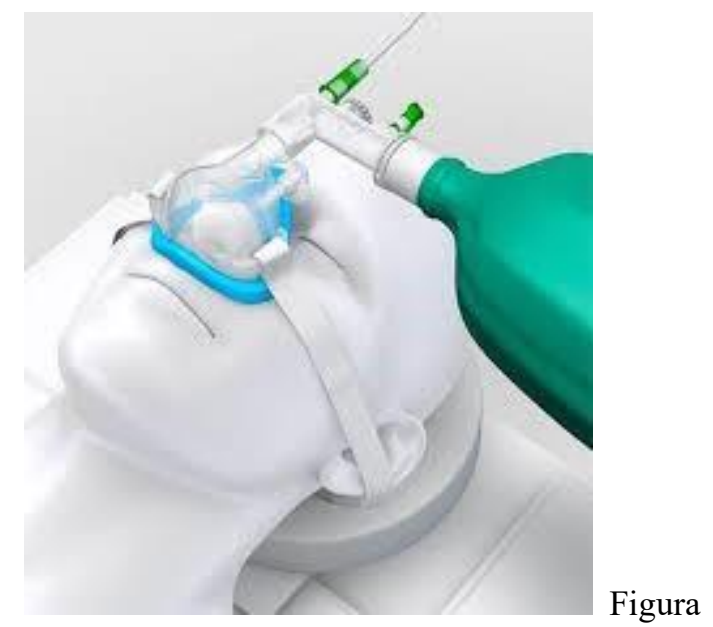

2. Sistema de PAP superNO2VA

El mecanismo de acción en caso de hipoxemia será similar a la CPAP usada para el manejo del Síndrome de ApneaHipopnea del sueño donde su eficacia está demostrada y cuenta con gran experiencia clínica tanto en adultos como en población pediátrica: al superar la presión de cierre y de colapso mantiene abierta la laringe y la vía aérea superior; esto a su vez permite la expansión pulmonar y evita la formación de atelectasias, mejorando el intercambio de gas en un pulmón congestivo y además puede reducir la hiperinsuflación dinámica que suele presentarse en casos de hiperreactividad bronquial o distrés $(19,20)$.

El sistema consiste en una máscara de ventilación que crea un sello cuando se coloca sobre la nariz del paciente para dirigir el flujo de aire y/u oxígeno a las vías respiratorias superiores durante todo el proceso respiratorio permitiendo mantener permeable la vía aérea $\mathrm{y}$ evitando su colapso. Incluye un set satélite que permite utilizar el dispositivo en trasporte de pacientes y en áreas del hospital sin disponibilidad de ventiladores.

La presión positiva en la vía aérea (PAP) nasal con superNO2VA permite aplicación de presión positiva en la vía aérea $\sin$ la necesidad de un equipo y personal especializado, es barato con respecto a otros dispositivos lo que ahorra costes y puede mejorar la oxigenación y la ventilación al mantener permeable la vía aérea $\mathrm{y}$ minimizar el efecto shunt y la alteración de la relación ventilación-perfusión típicas del distrés $(21,22)$.

Según su ficha técnica el sistema ha sido diseñado para:

- Preoxigenación efectiva y oxigenación apneica en diversas situaciones que precisan sedación.

- Aliviar la obstrucción de las vías aéreas superiores causada por disminución del nivel de conciencia.

- Mantener la vía aérea permeable y la ventilación.

- Realizar ventilación de emergencia.

- Facilitar el acceso para procedimientos intraorales $\mathrm{o}$ digestivos.

Se compone de la mascarilla (tamaño mediano y grande) con membrana de sellado del puente nasal, puerto de conexión al circuito de $15 \mathrm{~mm}$, bolsa reservorio de 2 , válvula APL con agujero de escape para limitar la presión, línea de conexión a la fuente de gas fresco, puerto de oxígeno suplementario que permite aumentar el suministro de flujo, tubo adicional para conexión de manómetro y correa de sujeción de velcro para ajuste craneofacial.

\section{Reporte de casos clínicos}

\section{Caso clínico $\mathbf{N}^{\circ} 1$.}

Varón de 83 años, ASA IV, con comorbilidad importante: EPOC con OCD, ICC, DM, IRC estadio III (EPOC (Enfermedad pulmonar obstructiva crónica), OCD (oxígeno crónico domiciliario), ICC (insuficiencia cardiaca congestiva), DM (Diabetes 
Mellitus), DL (Dislipemia), IRC (insuficiencia Renal crónica), CPAP (Presión positiva continua en la Vía aérea). Ingresado por cuadro de insuficiencia respiratoria global secundario a Neumonía por Coronavirus. El paciente no es candidato a ingreso en UCI por su poca probabilidad de supervivencia a largo plazo dada su edad y comorbilidad. Lleva tres días ingresado $\mathrm{y}$ ha empeorado respiratoriamente de manera progresiva a pesar de conseguir optimizar el manejo de sus patologías de base; está con deterioro del nivel de conciencia, disnea y taquipnea con 30 respiraciones por minuto, SatO2: $88 \%$ que no mejora al pasar de gafas nasales a VMK al 50\%; se intenta aumentar la oxigenación a través de una bolsa reservorio pero la Sat.O2 apenas mejora ligeramente a $90 \%$ y persiste con importante trabajo respiratorio $\mathrm{e}$ insuficiencia respiratoria global en la gasometría ( $\mathrm{pH}:$ 7,27. $\mathrm{PcO} 2: 81$ $\mathrm{mmHg}$. PO2 mmHg : 53. $\mathrm{HcO} 3: 37$ milimoles / lt). Ante la carencia importante de otros dispositivos de CPAP en ese momento decidimos aplicar PAP nasal con el dispositivo SuperNO2VA Talla L a $15 \quad 1$. y obteniendo un buen sellado; la saturación de oxígeno comienza a mejorar de manera inmediata y tras 2 horas de uso la clínica del paciente también mejora, está más despierto, con menos disnea y taquipnea $(20 \mathrm{rpm})$ y la SatO2 aumenta hasta 97\%. En la gasometría se confirma dicha mejoría: $\mathrm{pH}:$ 7,33. PcO2: $73 \mathrm{mmHg}$. PO2: 78mmHg, HcO3: 36 milimoles / 1t. El paciente evoluciona favorablemente $\mathrm{y}$ tras 72 horas alternando la PAP nasal con el VMK es posible el manejo de la oxigenación con gafas nasales (GN) convencionales a $31 / \mathrm{min}$. Finalmente es dado de alta a su domicilio tras 10 días de ingreso.

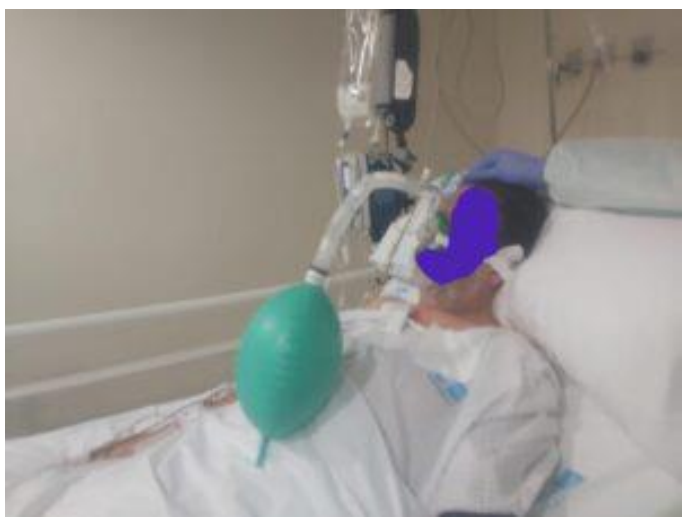

Figura 3. Adaptación del dispositivo

\section{Caso clínico $\mathbf{N}^{\circ} 2$.}

Varón de 62 años, ASA II, con antecedentes de EPOC grado II por tabaquismo y obesidad moderada con IMC de 34. Ingresado en la Unidad de Cuidados Intermedios Respiratorios (UCRI) procedente de la UCI donde estuvo ingresado 10 días por neumonía grave por coronavirus; precisó 7 días de ventilación mecánica invasiva.

El paciente se encuentra con agitación psicomotora, refiere disnea moderada y sed intensa con gran sequedad de mucosas; presenta taquipnea de $34 \mathrm{rpm}$ y Sat.O2 de 91\% con VMK al 50\%. La gasometría informa de hipercapnia e hipoxemia moderadas ( $\mathrm{pH}: 7,32$. $\mathrm{PcO} 2$ : 65mmHg PO2: 61 mmHg. HcO3: 32 milimoles / 1t). Tras sedación ligera con perfusión de dexmedetomidina a 0,6 $\mathrm{mcg} / \mathrm{kg} / \mathrm{h}$ se decide aplicar PAP nasal con el dispositivo SuperNO2VA a $15 \mathrm{lt}$ y conectar el puerto de oxígeno suplementario al aquapack de la pared para intentar humidificar un poco el flujo. Tras $1 \mathrm{~h}$ de soporte el paciente mejora notablemente, está más tranquilo, menos taquipneico $(20 \mathrm{rpm})$, satura $98 \%$ y ha podido beber sorbos de agua para paliar la sed. La gasomatría a las $2 \mathrm{~h}$ confirma la mejoría ( $\mathrm{pH}: 7,35$. PcO2: $55 \mathrm{mmHg}$. $\mathrm{PO} 2: 93 \mathrm{mmHg}$, HcO3: 30 milimoles / lt). Tras $6 \mathrm{~h} \mathrm{de}$ terapia el paciente presenta nuevamente un cuadro de agitación importante que impide continuar con el soporte, se 
quita continuamente la PAP y no es posible conseguir adaptarle a pesar de aumentar la sedación; el paciente presenta un notable empeoramiento respiratorio que finalmente requiere intubación y traslado de nuevo a UCI; allí permanece ingresado durante 15 días por un destete respiratorio complicado debido a una sobreinfección respiratoria bacteriana por Staphylococus aureus. Finalmente el paciente es destetado de nuevo y se realiza soporte postextubación con gafas nasales de alto flujo (GNAF) ya disponibles y tras 5 días de nuevo en UCRI se traslada planta y posteriormente dado de alta a su domicilio 7 días después.

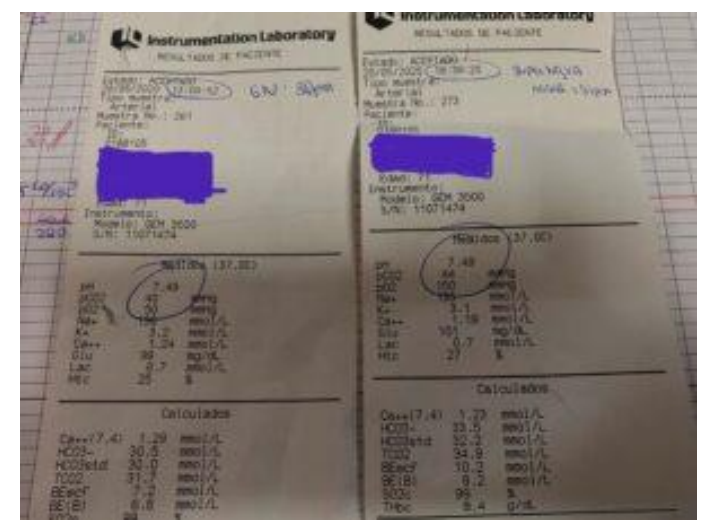

Figura 4. Gasometría arterial que muestra la mejoría en la oxigenación tras 4 h de terapia.

\section{Caso clínico $\mathrm{N}^{0} 3$}

Mujer de 71 años, ASA III, con antecedente de HTA, DL y portadora de valvuloplastia Mitral implantada hace 12 años por enfermedad valvular reumática. Consulta a urgencias por cuadro de malestar general y disnea progresiva en los últimos días; en la radiografía de tórax se observa cardiomegalia importante y derrame pleural bilateral; se le realiza ecocardiografía transtorácica que informa de una función globalmente reducida, insuficiencia tricuspídea libre e imágenes en la válvula mitral sugerentes de trombos. Se le realiza PCR para Coronavirus en el contexto de pandemia que es negativa $y$ se programa para intervención quirúrgica urgente; el protocolo anestésico y la técnica quirúrgica se realizan de manera habitual; tras la cardiotomía se procede a implantar una válvula tricuspídea Carbonics $\mathrm{N}^{\circ} 29$; en la válvula mitral no se objetiva trombos, sólo la presencia de abundante "pannus" en la base del anillo valvular, pero los discos estás limpios y se mueven de manera adecuada, por lo que se decide no cambiarla y se limpia el materia de la base, reforzando las suturas . Para la salida de la circulación extracorpórea precisa dosis altas de inotrópicos $\mathrm{y}$ vasoactivos (Dobutamina,

Norepinefrina) implantándose

finalmente un Balón intraórtico de contrapulsación (BIAC).

En la UCI el postoperatorio inmediato transcurre sin incidencias y se extuba tras 12 horas, con gran reducción de fármacos y a las 24 horas se retira el BIAC y los drenajes mediastínicos. Posteriormente la paciente presenta importante desaturación e hipoxemia a pesar de no tener disnea y sólo mínima taquipnea; se le realiza radiografía de tórax donde se visualiza mínimo derrame pleural bilateral y marcado componente atelectásico sobre todo en base derecha. La SatO2 se mantiene en $87 \%$ con GN y apenas sube a $89 \%$ con VMK al 50\%. En Los GA pH: 7,49 mmHg. $\mathrm{PcO} 2: 40 \mathrm{mmHg}$. PO2: 50 mmHg . HcO3: 30 milimoles / 1t. Decidimos aplicar PAP nasal con SuperNO2VA a 15 1. y la mejoría de la saturación es inmediata hasta llegar a $99 \%$. Tras 4 h la gasometría confirma la mejoría ( $\mathrm{pH}$ : 7,49. PcO2: $44 \mathrm{mmHg}$. PO2: 150 mmHg, HcO3: 33 milimoles / 1t).

Mantenemos la terapia durante $6 \mathrm{~h}$ continua y posteriormente la alternamos con periodos de GN convencionales hasta 48 horas que ya no presenta desaturación, tiene buena mecánica respiratoria y desaparece casi al 
completo las atelectasias y el derrame en la placa de tórax. Tras 4 dias en la UCI la paciente se traslada a planta para completar su recuperación postquirúrgica.

\section{Discusión}

Durante el periodo álgido de la epidemia en Madrid ocurrido entre la última quincena de Marzo y la primera de Abril, el hospital requirió de una rápida reorganización de sus instalaciones y una gran capacidad de respuesta de todo su personal; dada la abrumadora cantidad de ingresos de pacientes contagiados en situación de insuficiencia respiratoria de diversa gravedad en un periodo tan corto de tiempo fue necesaria una reestructuración a todos los niveles, desde al ámbito administrativo hasta la parte clínica, de servicios centrales y de todos los servicios de apoyo; casi todas las plantas fueron ocupadas con pacientes infectados, se redujo al mínimo el ingreso de pacientes por otra patologías y las consultas externas; se detuvo casi por completo la actividad quirúrgica programada dejando espacio y personal sólo para atender las emergencias, y fue necesario generar nuevos puestos de instalación de camas de críticos incluidos gran parte de los quirófanos del hospital, así como unidades de cuidados intermedios y respiratorios. En algún momento el hospital estuvo a más del $95 \%$ de su capacidad de ingresos, casi al borde del colapso. Fueron necesarias hasta 70 camas extra de cuidados críticos y al menos 40 de unidades de intermedios distribuidos por todo el centro para la atención de los casos más agraves. La respuesta a esta exigencia por parte del servicio de Anestesiología y Reanimación fue realmente encomiable, responsabilizándose de la atención de la mayoría de camas de críticos, y brindando soporte en unidades como las de cuidados respiratorios e intermedios e incluso en la planta de hospitalización, colaborando en la atención a todos los niveles con los diferentes servicios.

Esta situación desbordó la disponibilidad de medios en algún momento, lo que condicionó una escasez importante de material y nos vimos obligados a utilizar recursos de manera improvisada o para las tareas que habitualmente no estaban destinados (23); en el caso concreto del suministro de CPAP, en los días previos se nos había presentado la mascarilla SuperNO2VA como técnica de ayuda en el soporte respiratorio para las sedaciones en endoscopias y diversos procedimientos diagnósticos $\mathrm{y}$ terapéuticos del ámbito anestésico perioperatorio $\mathrm{y}$ en áreas fuera de quirófano. Así que aunque carecíamos de experiencia en su uso y dada la falta de los dispositivos suficientes de SRNI decidimos utilizarla en algunos casos concretos como los mencionados y en un total de 15 pacientes en algún momento: 5 pacientes en planta, 7 pacientes en UCRI y 3 pacientes en UCI post extubación. En dos casos utilizamos el dispositivo combinado con el prono en paciente despierto y colaborador $(24,25)$.

Los criterios que seguimos para su uso fueron:

- No disponibilidad de otros dispositivos con los que habitualmente tenemos más experiencia (GNAF, Mascarillas para VMNI, circuitos de Mapleson tipo C).

- Pacientes en planta con cuadro de insuficiencia respiratoria moderada, con persistencia de la desaturación a pesar de VMK o bolsa reservorio con el objetivo de mejorar la oxigenación, evitar la progresión de la hipoxemia y la necesidad de intubación endotraqueal. De tres casos con 
este objetivo, 2 fueron exitosos y el otro terminó requiriendo IOT y traslado a UCI.

- Pacientes en planta que por edad y/o coomorbilidad importante no tenían criterios de ingreso en UCI. De los dos casos con este objetivo, uno fue exitoso (caso clínico $\mathrm{N}^{\mathrm{o}} 1$ ) y el otro fracasó terminando con el fallecimiento del paciente.

- Pacientes en unidades de cuidados respiratorios intermedios, antes o después de su paso por UCI. De los 7 casos utilizados, 5 fueron exitosos y dos terminaron en fracaso (uno de ellos el caso clínico $\mathrm{N}^{\mathrm{o}} 2$, en el que luego se confirmó una sobreinfección respiratoria). De los casos exitosos en dos se combinó con el prono.

- Soporte respiratorio postextubación y como mecanismo para evitar la reintubación; de tres casos utilizado el dispositivo con este objetivo, los tres fueron exitosos (caso clínico $\mathrm{N}^{\circ} 3$ ).

Las ventajas que pudimos valorar del dispositivo son:

- Es sencillo y muy fácil de montar.

- Son solo dos tamaños, así que la selección del más adecuado para cada caso es fácil.

- Se puede usar en diferentes áreas del hospital, pues sólo se necesita q haya un caudalímetro de oxígeno. La versatilidad de la conexión del sistema permite incluso adaptarle un sistema de doble rama y conectarse a un respirador convencional.

- Se puede usar para el traslado del paciente entre diferentes áreas, conectado a una botella de oxígeno.
- Suficiente con 1 caudalímetro de 15 1. (llena bien la bolsa siempre que haya un buen sellado nasal). Se puede ampliar el flujo hasta 30 1. conectando otra línea de $\mathrm{O} 2$ al puerto accesorio, consiguiendo así general alto flujo.

- Al ser un sistema de PAP nasal exclusiva deja libre la boca del paciente, por lo que puede hablar y comunicarse mejor, puede beber líquidos $\mathrm{y}$ en general está más confortables. No refieren la sensación de claustrofobia q experimentan con otras mascarillas (oronasales, totalface, hellmet, Decathlón, etc).

Entre las desventajas:

- Carecemos aún de experiencia con el uso del dispositivo.

- Al disponer sólo de dos tamaños, en algunos pacientes no encontramos la interfase más adecuada.

- Precisa un buen llenado de la bolsa y ajuste de la válvula APL para garantizar la CPAP.

- Un buen sellado se consigue ajustando bien la correa de sujeción cruzando por en medio de las orejas lo que a veces general molestia (poner gasitas de protección para evitar el daño del cartílago).

- No sabemos con certeza la cantidad de CPAP que se está suministrando en cada momento. Si bien se dispone de puerto para conectar manómetro.

- A veces es necesario ejercer mucha presión con el velcro para poder conseguir un buen sellado, lo que resulta incómodo y lesivo para el paciente.

- Con el uso prolongado ejerce presión sobre el dorso nasal y eso reduce la tolerancia; ha sido 
la principal queja de los pacientes.

- Es recomendable un buen nivel de conciencia y a poder ser que el mismo paciente ayude en el ajuste de la mascarilla.

- Si el paciente lleva sonda nasogástrica es muy difícil conseguir un buen sellado.

- Requiere una respiración nasal para que el dispositivo cumpla su objetivo; la taquipnea importante y la respiración oral son muy propensos al fracaso.

\section{Conclusión}

La epidemia desatada por la infección por coronavirus ha desbordado prácticamente todos los sistemas sanitarios del mundo y ha puesto en evidencia una falta de preparación del modelo de atención para abordar situaciones de esta envergadura; Madrid ha sido una de las regiones más afectadas en España y la práctica totalidad de sus hospitales se vieron en algún momento superados y con falta de medios adecuados y suficientes tanto para la atención de los pacientes como para la protección de los sanitarios; dada su amplia experiencia en el manejo de la vía aérea, la ventilación mecánica y los diferentes dispositivos existentes para ello, así como la destreza probada en los cuidados críticos médicos y perioperatorios, el servicio de Anestesiología y Reanimación ha sido imprescindible en la diversificación y manejo de unidades de críticos y cuidados respiratorios en todas las áreas del hospital; la más que conocida capacidad de adaptación e improvisación en momentos de crisis de éstos especialistas ha permitido utilizar todas las herramientas disponibles en cada momento para conseguir los mejores resultados posibles a la hora de enfrentar la gran demanda de pacientes graves. Si bien la superNO2VA no es un dispositivo desarrollado en un principio para el manejo de la insuficiencia respiratoria o el distrés, su mecanismo de funcionamiento nos permitió en un momento de precariedad de recursos utilizarlo para mejorar la situación clínica en algunos pacientes, la mayoría de veces con buenos resultados. Puede ser una alternativa a otras dispositivos a la hora de tratar pacientes con insuficiencia respiratoria de diversa etiología, pero son necesarios más estudios y desde luego probar su eficacia en el tiempo para determinar realmente su potencial alcance en la práctica clínica habitual.

\section{Bibliografía}

1. Centers for Disease Control and Prevention. 2019 Novel coronavirus, Wuhan, China. Information for Healthcare Professionals. https://www.cdc.gov/coronavirus/ 2019-nCoV/hcp/index.html (Accessed on February 14, 2020).

2. World Health Organization. Novel Coronavirus (2019-nCoV) technical guidance. https://www.who.int/emergencies/dis eases/novel-coronavirus-2019/technicalguidance (Accessed on February 14, 2020).

3. Ministerio de Sanidad. Manejo clínico de pacientes con enfermedad por el nuevo coronavirus (COVID-19). Disponible en: https://www.mscbs.gob.es/profesionales/sal udPublica/ccayes/alertasActual/nCov-

China/documentos Protocolo_manejo_clinico_C OVID-19.pd.

4. Mosites E, Parker EM, Clarke KEN, et al. Assessment of SARS-CoV-2 Infection Prevalence in Homeless Shelters - Four U.S. Cities, March 27-April 15, 2020. MMWR Morb Mortal Wkly Rep 2020; 69:521.

5. European Centre for Disease Prevention and Control. Infection prevention and control for the care of patients with $2019-\mathrm{nCoV}$ in healthcare settings [Internet]. 2020 feb. Disponible en: Infection prevention and control for the care of patients with 2019-nCoV in healthcare settings. (

6. Gorbalenya AE, Baker SC, Baric RS, et al. Severe acute respiratory syndrome-related coronavirus: The species and its viruses $-\mathrm{a}$ 
statement of the Coronavirus Study Group. bioRxiv

2020. https://www.biorxiv.org/content/10.1101/ 2020.02.07.937862v1 (Accessed on February $12,2020)$.

7. Zhou F, Yu T, Du R, et al. Clinical course and risk factors for mortality of adult inpatients with COVID-19 in Wuhan, China: a retrospective cohort study. Lancet 2020; 395:1054. (HTML)

8. Ñamendys-Silva SA. Respiratory support for patients with COVID-19 infection. Lancet Respir Med 2020; 8:e18.

9. Gattinoni L, Chiumello D, Caironi P, et al. COVID-19 pneumonia: different respiratory treatments for different phenotypes? Intensive Care Med 2020.

10. Gattinoni L, Quintel M, Marini JJ. "Less is More" in mechanical ventilation. Intensive Care Med 2020; 46:780.

11. Gomez C, Rodríguez O, Luján M, Egea C, Masa JF, García , et al. Clinical Consensus Recommendations Regarding Non-Invasive Respiratory Support in the Adult Patient with Acute Respiratory Failure Secondary to SARSCoV-2 infection. Revista Española de Anestesiología y Reanimación, Vol. 67. Núm. 5.páginas 261-270 (Mayo 2020). (PubMed)

12. Ding L, Wang L, Ma W, He H. Efficacy and safety of early prone positioning combined with HFNC or NIV in moderate to severe ARDS: a multi-center prospective cohort study. Crit Care 2020; 24:28.

13. Leung $\mathrm{CCH}$, Joynt GM, Gomersall CD, et al. Comparison of high-flow nasal cannula versus oxygen face mask for environmental bacterial contamination in critically ill pneumonia patients: a randomized controlled crossover trial. J Hosp Infect 2019; 101:84.

14. Hui DS, Chan MT, Chow B. Aerosol dispersion during various respiratory therapies: a risk assessment model of nosocomial infection to health care workers. Hong Kong Med J 2014; 20 Suppl 4:9.

15. Cheung JC, Ho LT, Cheng JV, et al. Staff safety during emergency airway management for COVID-19 in Hong Kong. Lancet Respir Med 2020; 8:e19.
16. Ficha Técnica SuperNO2VA dispositivo de ventilación nasal PAP. Vyaire Medical. ( $\underline{\text { HTML) }}$

17. Cataldo S, Pedro M, Straker T, Osborn I. The superNO2VA to treat upper airway obstruction and respiratory compromise after major head and neck surgery: a case report. Journal of Head and Neck Anesthesia vol. 3 No2; Mayo 2019. (HTML)

18. Bastien A, Pedro M, Cataldo S. Treating oxygen failure in angioedema: a case series employing noninvasive nasal positive pressure with the SuperNO2VA device. Journal of Head and Neck Anesthesia Vol.3 No2; Mayo 2019. (HTML)

19. Ghebremichael, Sembar, et al. Evaluation of SuperNO2VA mask technology in a clinical setting: A pilot study. Trends in anesthesia and critical care 2017; 16:64-61. (HTML)

20. Xu, Zeping, Bai, Yiping, Jiang, Yandong, Koffi, Kia. Comparison of a simplified nasal continuous positive airways pressure device with nasal cannula in obese patients undergoing colonoscopy during deep sedation. A randomized clinical trial. European Journal of Anesthesiology 2019; 36:1-8 (ㅍML)

21. Dimou F, Huynh S, Dakin G, Pomp A, Turnbull Z, Samuels J, et al. Nasal positive pressure with the SuperNO2VA ${ }^{\mathrm{TM}}$ device decreases sedation-related hypoxemia during pre-bariatric surgery EGD. Surgical endoscopy (2019): 1-5. (PubMed)

22. Patil SP, Ayappa IA, Caples SM, et al. Treatment of Adult Obstructive Sleep Apnea With Positive Airway Pressure: An American Academy of Sleep Medicine Systematic Review, Meta-Analysis, and GRADE Assessment. J Clin Sleep Med 2019; 15:301.

23. Y. Ji, Z. Ma, M.P. Peppelenbosch, Q. Pan. Potential association between COVID-19 mortality and health-care resource availability.Lancet Glob Health., (2020).

24. Scaravilli V, Grasselli G, Castagna L, et al. Prone positioning improves oxygenation in spontaneously breathing nonintubated patients with hypoxemic acute respiratory failure: A retrospective study. J Crit Care 2015; 30:1390.

25. Pérez-Nieto OR, Guerrero-Gutiérrez MA, Deloya-Tomas E, Namendys-Silva SA. Prone positioning combined with high-flow nasal 
cannula in severe noninfectious ARDS. Crit Care 2020; 24:114.
Correspondencia al autor

Ramiro Adrián Mira Betancur rubenferrerasvega@hotmail.com

FEA Servicio de Anestesiología y Reanimación, Hospital Universitario Ramón y Cajal, Madrid.

Aceptado para el blog en junio de 2020 